\title{
Jean Copans. Georges Balandier. Un anthropologue en première ligne
}

\section{Pascale Moity-Maïzi}

\section{OpenEdition}

\section{Journals}

Édition électronique

URL : http://journals.openedition.org/anthropodev/453

DOI : 10.4000 /anthropodev.453

ISSN : 2553-1719

Éditeur

APAD - Association pour l'anthropologie du changement social et du développement

Édition imprimée

Date de publication : 1 juillet 2016

Pagination : 191-193

ISBN : 797-10-93476-03-2

ISSN : 2276-2019

Référence électronique

Pascale Moity-Maïzi, « Jean Copans. Georges Balandier. Un anthropologue en première ligne »,

Anthropologie \& développement [En ligne], 44 | 2016, mis en ligne le 01 décembre 2016, consulté le 24 septembre 2020. URL : http://journals.openedition.org/anthropodev/453 ; DOI : https://doi.org/ 10.4000/anthropodev.453

La revue Anthropologie \& développement est mise à disposition selon les termes de la Licence Creative Commons Attribution 4.0 International. 


\section{Georges Balandier Un anthropologue en première ligne \\ Jean Copans, \\ Paris, PUF, 2014, 313 p.}

Pascale Moity-Maïzi

Présenté comme un " essai d'histoire intellectuelle » par son auteur, cet ouvrage est à la fois le récit détaillé d'une trajectoire de vie, l'exégèse d'une œuvre, un manuel pédagogique et même un plaidoyer pour repenser les sciences sociales.

L'ouvrage est structuré en sept chapitres précédés d'un prologue qui annonce une approche en " six vies » de G. Balandier, caractérisant l'œuvre en même temps que son auteur. On comprend vite que ces vies se répartissent en deux grands moments, la période africaniste, dédiée aux situations coloniales et post-coloniales, puis la période durant laquelle G. Balandier tisse une relation de plus en plus complexe avec le concept ou les questions de développement. Tout au long de ce livre remarquablement écrit, J. Copans s'adresse directement au lecteur et joue de postures différentes à travers sa relecture des écrits de G. Balandier : il nous propose tour à tour un retour réflexif sur la sociologie et l'anthropologie (G. Balandier n'ayant jamais su se définir), une exégèse minutieuse de certains ouvrages déterminants dans l'histoire de ce champ disciplinaire, une traque de l'homme dans l'œuvre, une critique littéraire de celui qui se rêvait parfois romancier. Jean Copans ne reconstitue pas une trame chronologique pour présenter les multiples facettes qui animent les travaux de G. Balandier : il traverse les temporalités de ce dernier selon ces six « vies » qui se croisent en différentes parties.

Pour ce faire, tous les textes de G. Balandier, quels qu'ils soient, sont relus en étant systématiquement replacés dans leurs contextes (politiques, intellectuels), et véritablement revivifiés par les éclairages nouveaux que propose Jean Copans. Certains textes sont largement cités et précisément contextualisés car ce sont les documents "d'une certaine histoire intellectuelle et scientifique » (p.82), dans laquelle G. Balandier construit ses vies. La première est celle de " l'africanisation » où l'on redécouvre la conjoncture politique et institutionnelle de la colonisation et l'évolution de G. Balandier comme ethnologue-sociologue chargé de recherches commanditées. La seconde vie, c'est celle de la " situation coloniale ", entendue comme conceptualisation dynamique des phénomènes sociopolitiques en Afrique, fondatrice pour la réflexion ultérieure de G. Balandier sur le sous-développement et plus largement pour l'anthropologie politique française; période déterminante aussi en ce qu'elle oblige $\mathrm{G}$. Balandier à se positionner parmi ses pairs. La troisième vie correspond à la période où "son identité africaniste cède provisoirement la 
place à celle du spécialiste du changement social et de la modernisation avant de se transformer en celle du changement socioculturel et de la modernité politique à partir des années 1960 »(p.112) : l'approche d'une situation coloniale évolue vers l'analyse des dépendances puis du sous-développement, sociologie et anthropologie étant ici nécessaires pour saisir des réalités inédites, dynamiques et complexes. Cette troisième vie est l'occasion pour J. Copans de revenir sur l'histoire de quelques notions aujourd'hui banalisées ou déjà oubliées, telles que "TiersMonde ", " sous-développement » et " mouvement social ». G. Balandier analyse à travers cette dernière non pas tant les situations de dépendances que les mouvements socioculturels, politiques et religieux qui s'y opposent (p.123), et à travers eux la montée des nationalismes ${ }^{1}$. C'est aussi à partir de cette vie-là que Jean Copans souligne le glissement qui s'opère : son éloignement vis-à-vis du sousdéveloppement conduirait $\mathrm{G}$. Balandier à le reformuler en termes de tradition et de modernité (p.134) et à déplacer le regard du Sud vers le Nord. II se lance alors " dans l'exploration de l'inédit, de l'inattendu, des nouveaux Nouveaux Mondes » (p.141). La quatrième vie qualifiée de " seconde carrière paradigmatique » (p.143) nous mène dans une pensée ardue, sans doute parce que jamais personne n'avait encore analysé cette période complexe, moins connue aussi, de l'œuvre de G. Balandier depuis 1985. Le travail de J. Copans est ici totalement inédit, plus critique aussi, soulignant les oublis, les limites de l'engagement ou de l'analyse de G. Balandier. Les deux vies suivantes que nous synthétise J. Copans, révèlent un auteur qui doute non seulement des institutions régulatrices de nos sociétés mais aussi de l'anthropologie désormais impuissante à saisir la complexité sociale et qui semble " avoir perdu ses vertus de détournement » (p.169). Le pessimisme croissant de G. Balandier est presque morbide, c'est un peu comme s'il s'effaçait peu à peu pour laisser la place à ses élèves. Jean Copans, en l'occurrence, développe ici sa propre lecture de toute l'anthropologie politique de Georges Balandier, dans ses méandres et contradictions, comme s'il voulait lui donner un regain de sens. L'ancien élève se positionne désormais comme traducteur et porte-parole de G. Balandier, et parachève là un travail de conceptualisation nécessaire ne serait-ce que pour pouvoir affirmer sa propre filiation intellectuelle (p.263) tout en exprimant sa distance critique progressivement. L'effet de ce travail est pluriel, mais il permet de souligner l'importance intellectuelle et historique de G. Balandier pour penser la complexité sociale et nos disciplines. Cela d'autant plus qu'il nous incite à réinventer les formes nouvelles d'une science sociale "dans le bas parce que c'est d'en bas qu'on voit le mieux les autres sans que ces derniers aient la malheureuse impression [...] que les sciences sociales continuent à les regarder de haut "

${ }^{1}$ C'est de toute évidence dans cette troisième vie que l'APAD a pris racine. Les pages 129 à 136 sont remarquables à cet égard. 
(p.295). La démarche de J. Copans qui consiste à rassembler les sources, à retrouver des contextes d'inspiration et d'influence scientifiques, ou même à se mettre à la place de G. Balandier puis à s'en faire porte-parole, est à la fois un procédé de dévoilement et d'enrôlement : il nous dévoile certes la logique de la pensée de G. Balandier, mais il nous restitue aussi l'histoire de la sociologie et de l'anthropologie française sur presque un siècle en essayant au passage de nous faire comprendre pourquoi G. Balandier ne s'est jamais clairement positionné sur l'une ou l'autre des disciplines, oscillant de l'une à l'autre selon les époques et les opportunités institutionnelles.

Cette minutieuse relecture de tous les documents fait littéralement revivre certains textes oubliés. J. Copans nous prend par la main pour nous guider dans la pensée complexe de G. Balandier, et pour nous révéler ou nous rappeler les éléments fondateurs d'une anthropologie politique du changement social telle qu'elle a été défendue par I'APAD. Par exemple le fait d'être situé " au carrefour de l'action pratique, prépolitique en un sens, et de la compréhension scientifique " (p.57), le fait de privilégier une approche du changement social plutôt que celle de l'organisation ou de la " modernisation » des sociétés africaines, sont quelques-uns des fondements historiques à l'origine de la création de I'APAD... Enfin, le regard novateur et particulièrement stimulant que nous propose J. Copans sur toute l'œuvre de G. Balandier n'a de cesse de mettre au jour des ingrédients et cadres structurants pour repenser notre actualité politique, celle de ce début de $\mathrm{XXI}^{\mathrm{e}}$ siècle, marquée par la multiplication et la globalisation des mouvements sociaux, par la montée des revendications identitaires dans des arènes internationales qui, pourtant, n'entraînent pas la fin des dominations.

Mais au fond pourquoi Jean Copans s'est-il engagé à rédiger un ouvrage sur Georges Balandier? On le comprend au fil de l'ouvrage : parce que G. Balandier est "à la fois un fils de l'Afrique et un citoyen du monde" (p.46), parce que les sciences sociales ne seraient pas ce qu'elles sont aujourd'hui " sans cette longue fidélité que G. Balandier a conservé à l'égard de son détour anthropologique et sociologique » (p.47), parce qu'enfin Jean Copans souhaite sans doute motiver les lecteurs d'aujourd'hui à revenir à des fondamentaux : il n'est pas possible, selon J. Copans, de "penser la société moderne mondiale, qui inclut [...] aussi bien la "situation coloniale" que les "nouveaux Nouveaux Mondes", sans l'imaginaire sociologique de G. Balandier » (p.188). Et pour ceux et celles qui n'auraient de Georges Balandier qu'un vague souvenir académique, cet ouvrage est nécessaire pour redécouvrir l'anthropologue qu'il a été et comprendre de quelle manière nous en sommes tous peu ou prou « imprégnés »! 